\title{
Packet Loss and Packet Delivery Evaluation Using Network Simulator for Multicast Enabled Network Mobility Management
}

\author{
Azana Hafizah Mohd Aman ${ }^{1}$, Aisha-Hassan A. Hashim², Huda Adibah Mohd \\ Ramli and Shayla Islam \\ Kulliyyah of Engineering, International Islamic University Malaysia, Jalan \\ Gombak 53100, Kuala Lumpur, Malaysia \\ 1azana05@yahoo.com,2aisha@iium.edu.my
}

\begin{abstract}
This paper investigates packet loss issue in multicast enabled network mobility management using network simulator. Network mobility management namely PMIPv6 has the capacity to reduce mobile IPv6 performance problems. But PMIPv6 basic architecture is limited to unicast communication. Therefore, this paper proposes PMIPv6 with context delivery and multicast fast reroute mechanisms to enable multicast services. In this paper, the configuration of multicast enabled PMIPv6 is done in network simulator called NS3. The aimed is to solve packet loss problem. This paper assumes that packet loss as the number of packets that are lost in a multicast communication. Packet loss is unescapable particularly in mobile multicast communication. It is a network system requirement to meet the threshold value of acceptable packet loss rate below than 5\%. The packet loss ratio and the packet delivery ratio are extracted from the trace-file produced by the simulation process in NS3 simulator. The analyses of the packet loss ratio and packet delivery ratio are hereby presented.
\end{abstract}

Keywords: NS3, Packet Loss, Packet Delivery, Mobile Multicast

\section{Introduction}

By year 2020, mobile data traffic will reach 30.6 exabytes of monthly global mobile data traffic [1-3]. $75 \%$ of the global mobile traffic will be video and $66 \%$ of devices will be connecting to IPv6 mobile network. In recent years, there are huge interest in mobile multicast IP development, nevertheless, until now there is no typical standard [4-5]. This paper presents an improvement in term of packet loss and packet delivery in order to provide high context delivery in multicast enabled network mobility management. The improvement is done via implementation of Proxy Mobile Ipv6 (PMIPv6) [7-8] with context delivery [9-11] and multicast fast rerouting [12]. This new implementation is called CTMFR. The aim is to improve packet loss and packet delivery performance. The analysis of context delivering is through NS3 simulator and benchmarked with the standard [13].

The outlined of this paper is as follows. Section 2 described related research in network mobility management development. While Section 3 illustrated the context delivery fast reroute implementation. Section 4 explained the simulation analysis. Section 5 , is the summary.

\section{Related Research}

The fundamental approach to deal with mobility in IPv6 is stated in MIPv6 [6]. Many mobile multicast methods amended the standard MIPv6 to enable multicast communication. The present mobility management are not generally accepted and with no effective flow to care for multicast communication [14]. Future Internet mobile multicast 
support must be able to provide 'close to optimal' performance with expected cost compliant to real-time data distribution [15].

Work in [16] implemented an effective handover for multicast subscribers. It delivers optimal delivery path for intra domain transfer by implementing an efficient handover multicast subscribers. While work in [17] proposed route optimization method to improve multicast traffic in PMIPv6, it focused on the implementation of multicast support on the MAGs. Work in [18] proposed a direct multicast routing with base solution for mobile multicast destination support in PMIPv6. The results outperform the benchmarked methods in terms of signaling cost.

Work in [19] enhanced the functionality of PMIPv6 by introducing a multicast enabled handover technique. The technique improves the performance by eliminating the false handover initiation. The obtained simulation results show a reduction in packet loss and handover latency which improves the handover performance. In [20], the implementation of a Proxy Fast MIPv6 was validated with actual deployment implementation. Work in [21] aimed to specifically study PMIPv6 mobility management protocol to investigate the performance of mobile handover that occur in the mobility protocol implementation. While in [22], it presented deployment for enabling distributed mobility management with IP multicast.

\section{Fast Reroute Content Delivery Implementation}

Improving PMIPv6 in order to allow multicast communication to pass through is essential. This is because PMIPv6 only allows unicast communication to pass through. This paper enables multicast via context delivery and multicast fast reroute mechanism to the standard network mobility management. This mechanism provides multicast services with high network performance in term of packet loss and packet delivery.

This mechanism provides the delivery of the multicast context in advanced. Therefore reduces unnecessary transmission after the handover. As for the fast reroute, it allows the existence of primary and secondary path for the multicast data delivery. This eliminates high packet loss problem.

By using this mechanism, the overall process needed to continue the multicast service after the handover is very minimal. This is because the context delivery is transmitted to the MAGs before the handover. Therefore, all the necessary data for the MN to join the multicast group is sent earlier. The $\mathrm{MN}$ received the multicast traffic directly after the $\mathrm{MN}$ transfers to the nMAG. The mobile node is not involved in sending the multicast data to the nMAG or LMA.

Figure 1 displays the basic network topology for the new mechanism. Figure 2 shows the context transfer process flow. Figure 3 illustrates the multicast fast reroute process flow. 


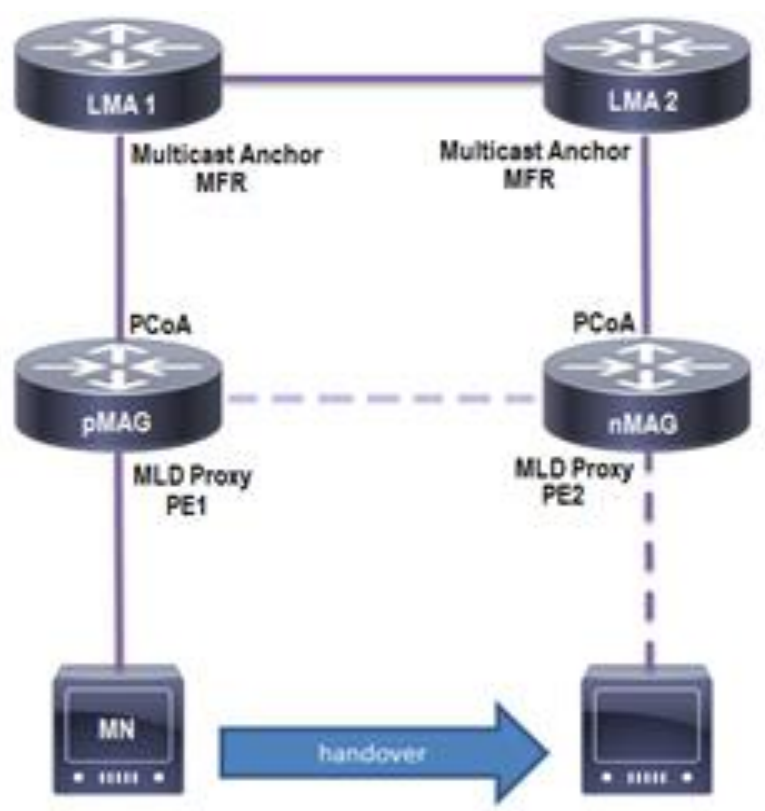

Figure 1. Basic Network Topology for the Implementation

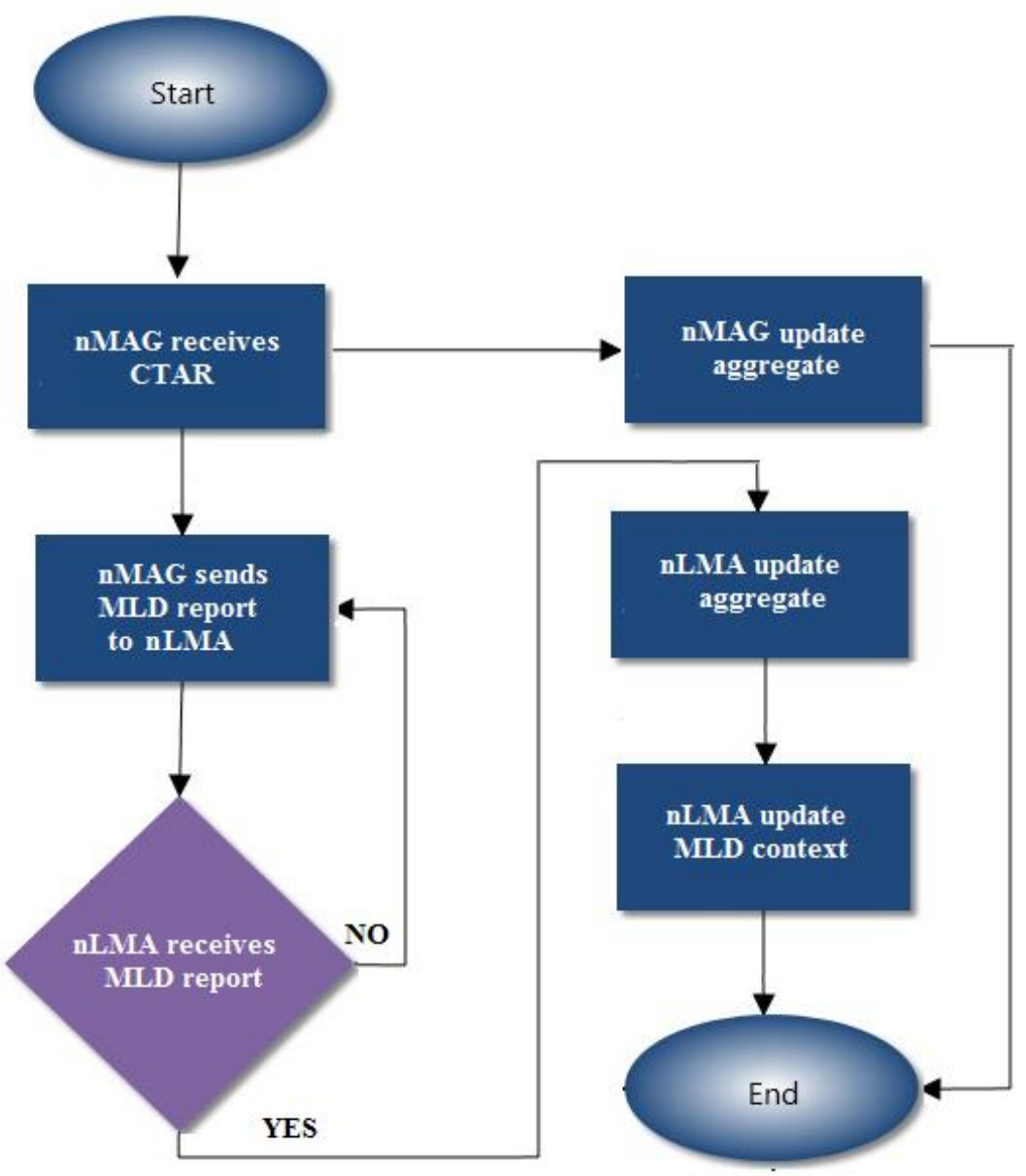

Figure 2. Context Transfer Process Flow 


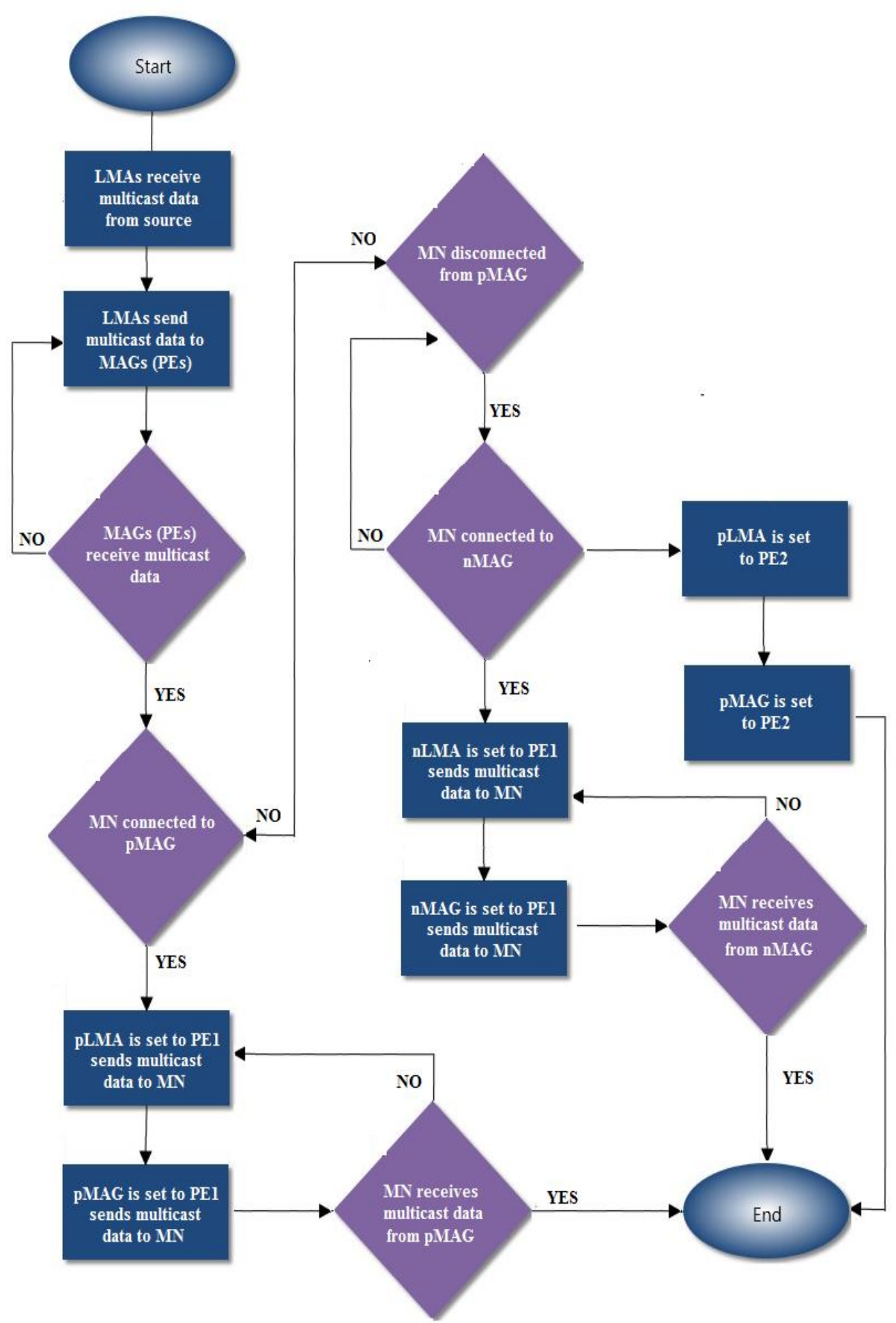

Figure 3. Multicast Fast Reroute Process Flow

Basically the NS3 is configured with PMIPv6 environment and User Datagram Protocol (UDP) as the traffics. Performance metric measured is packet loss and packet delivery. The results are collected in PCAP modules. NetAnim file is used for trace files processing written in XML extension to outcast the simulation as shown in Figure 4 and Figure 5. While Gnuplot is a portable command-line driven graphing utility used to display the results obtain in graphs as shown in Figure 6 to Figure 9. Figure 5 shows the 
NS3 simulation setup with 18 nodes. While Figure 5 shows part of the simulation. Table 1 shows the NS3 parameters.

\section{Table 1. Parameters for Simulation}

\begin{tabular}{|l|l|}
\hline \multicolumn{1}{|c|}{ Parameter } & \multicolumn{1}{c|}{ Values } \\
\hline Simulator & NS3.19 (Ubuntu 14.04) \\
\hline Simulation time & $100-500 \mathrm{~s}$ \\
\hline MN velocity & $20 \mathrm{n}-60 \mathrm{~m} / \mathrm{s}$ \\
\hline Bitrate & CBR \\
\hline No of nodes & 18 \\
\hline LMA-MAG Link Delay & $10 \mathrm{~ms}$ \\
\hline MAG-AP Link Delay & $2 \mathrm{~ms}$ \\
\hline Data Rate & $50 \mathrm{Mbps}$ \\
\hline
\end{tabular}

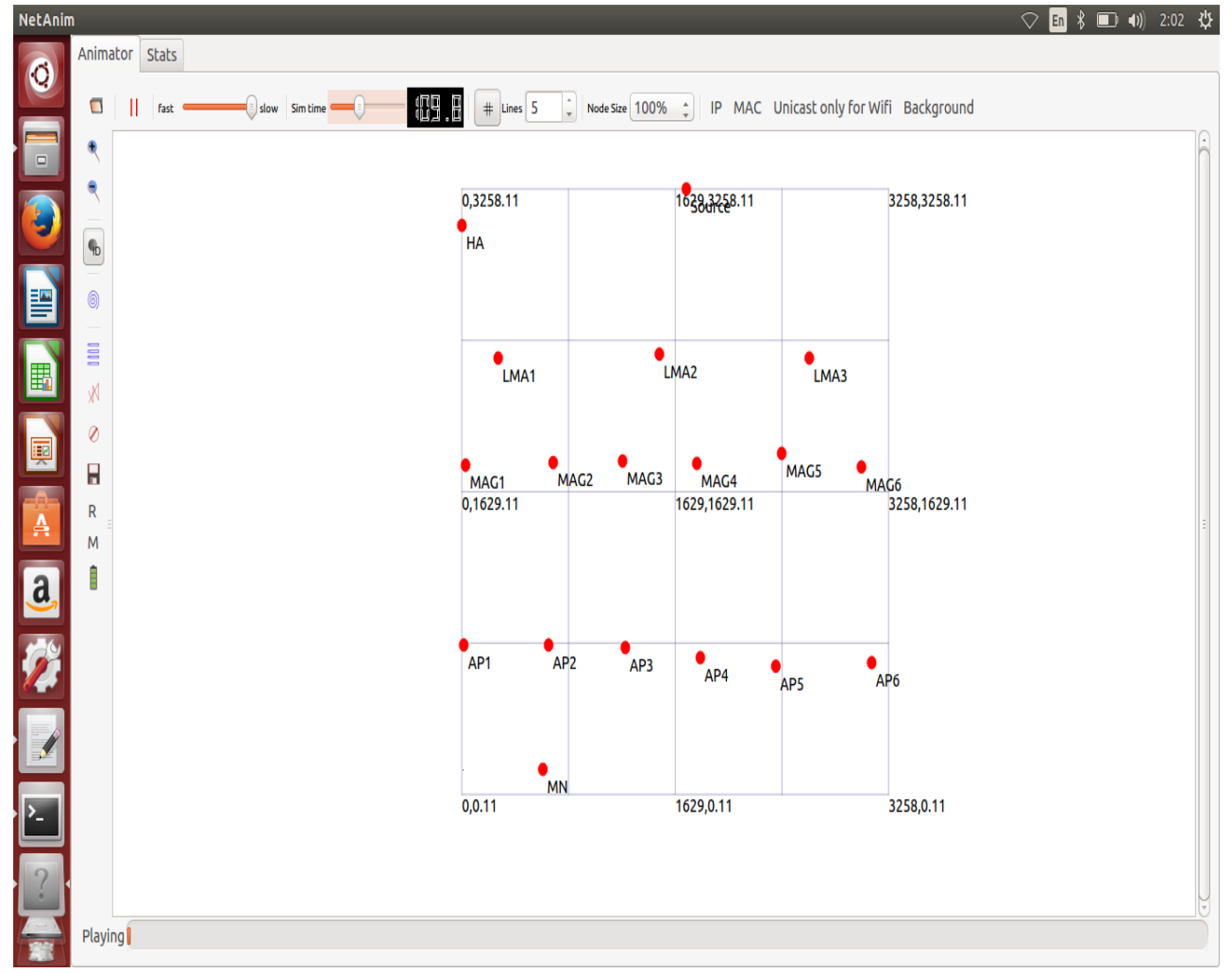

Figure 4. NetAnim PMIPv6 Topology 


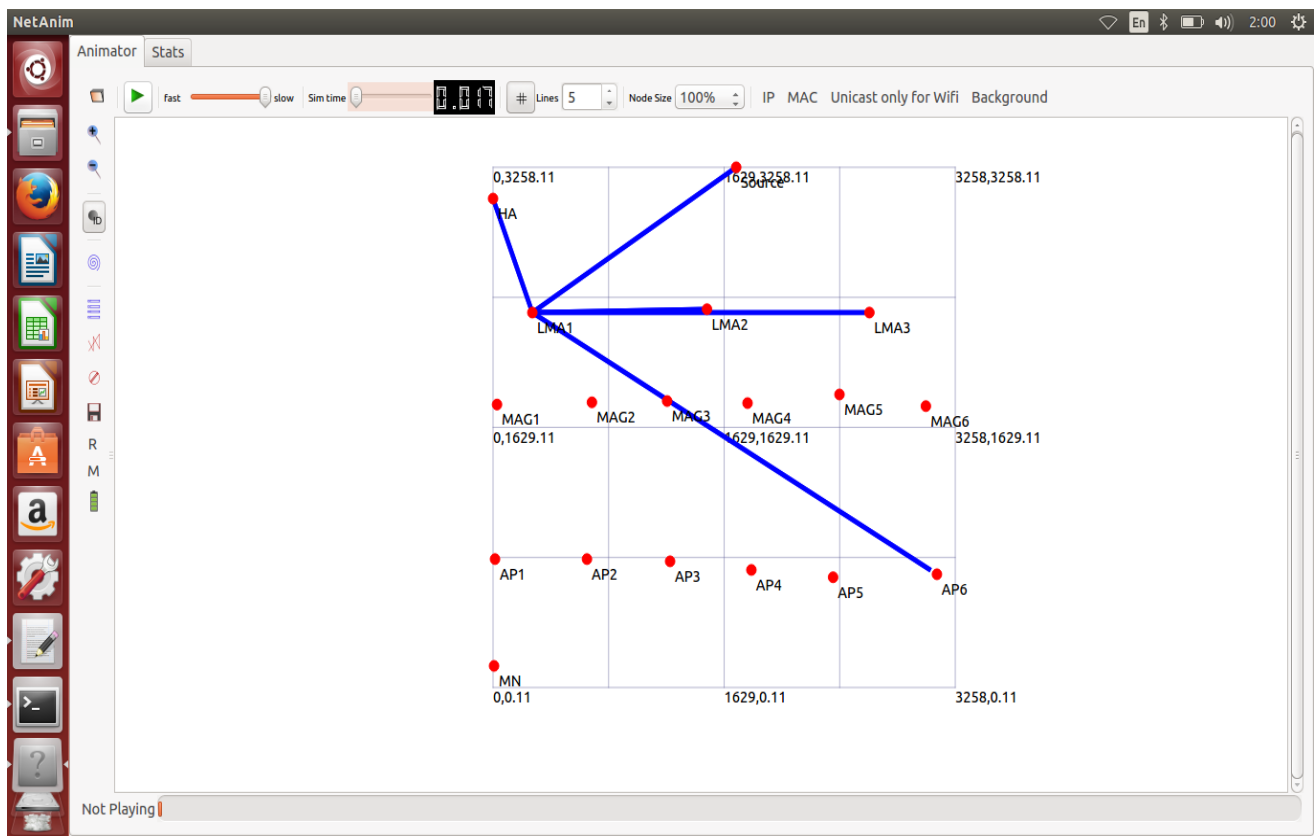

Figure 5. NetAnim PMIPv6 Simulation

\section{Simulation Analysis}

As the mobile node changes it location, it suffers from packet loss. It is high possibility that the new visiting network does not enable multicast causing rejection of all the multicast traffic. Due to this, multicast fast reroute and context delivery PMIPv6 is selected and implemented in NS3. The packet lost ratio results for NS3 is given in Figure 6 and Figure 7, with 500 number of maximum simulation time, with increase value of mobile node speed of $20 \mathrm{~m} / \mathrm{s}$ for each $100 \mathrm{~s}$ simulation time. From Figure 6 , it shows that the packet loss ratio increases as the simulation time and mobile node speed increases. It can be seen from Figure 7, that the implementation appears as low in comparison with high packet loss during handover in standard PMIPv6. On average the proposed method only faced not more than $3.7 \%$ packet lost ratio whereas the benchmarked faced more than $12 \%$ packet lost ratio. In other words, the CTMFR helps to minimize the packets loss ratio.

Figure 8 and Figure 9 shows the packet delivery ratio for the implementation and also for comparison with the standard benchmark. As shown the packet delivery ratio decreases as the simulation time and mobile node speed increases. However, the new implementation performs better than the benchmark. As can be seen the packet delivery ration is inversely proportional to the packet loss ratio. 


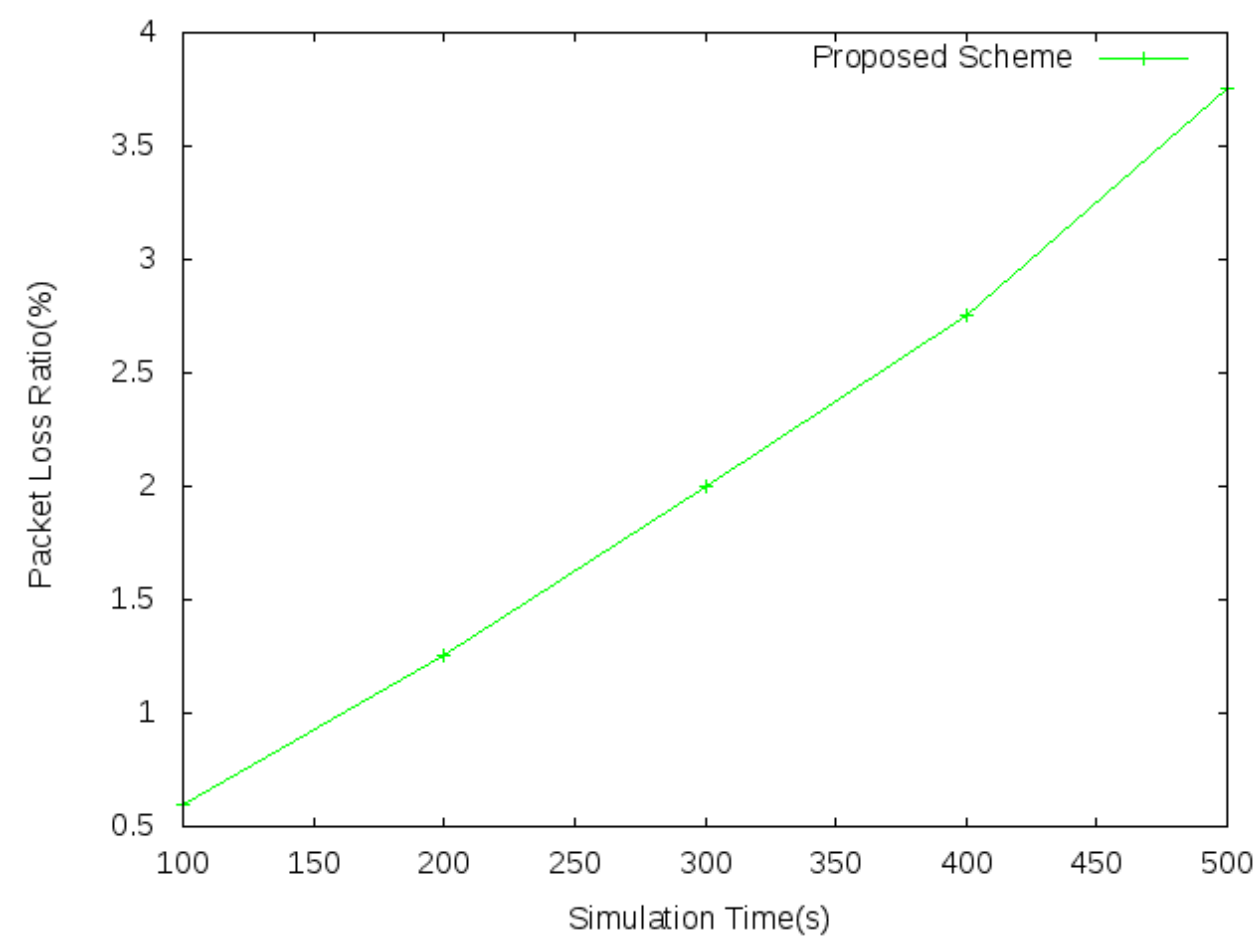

Figure 6. Packet Lost Ratio versus Simulation Time for the Proposed Implementation

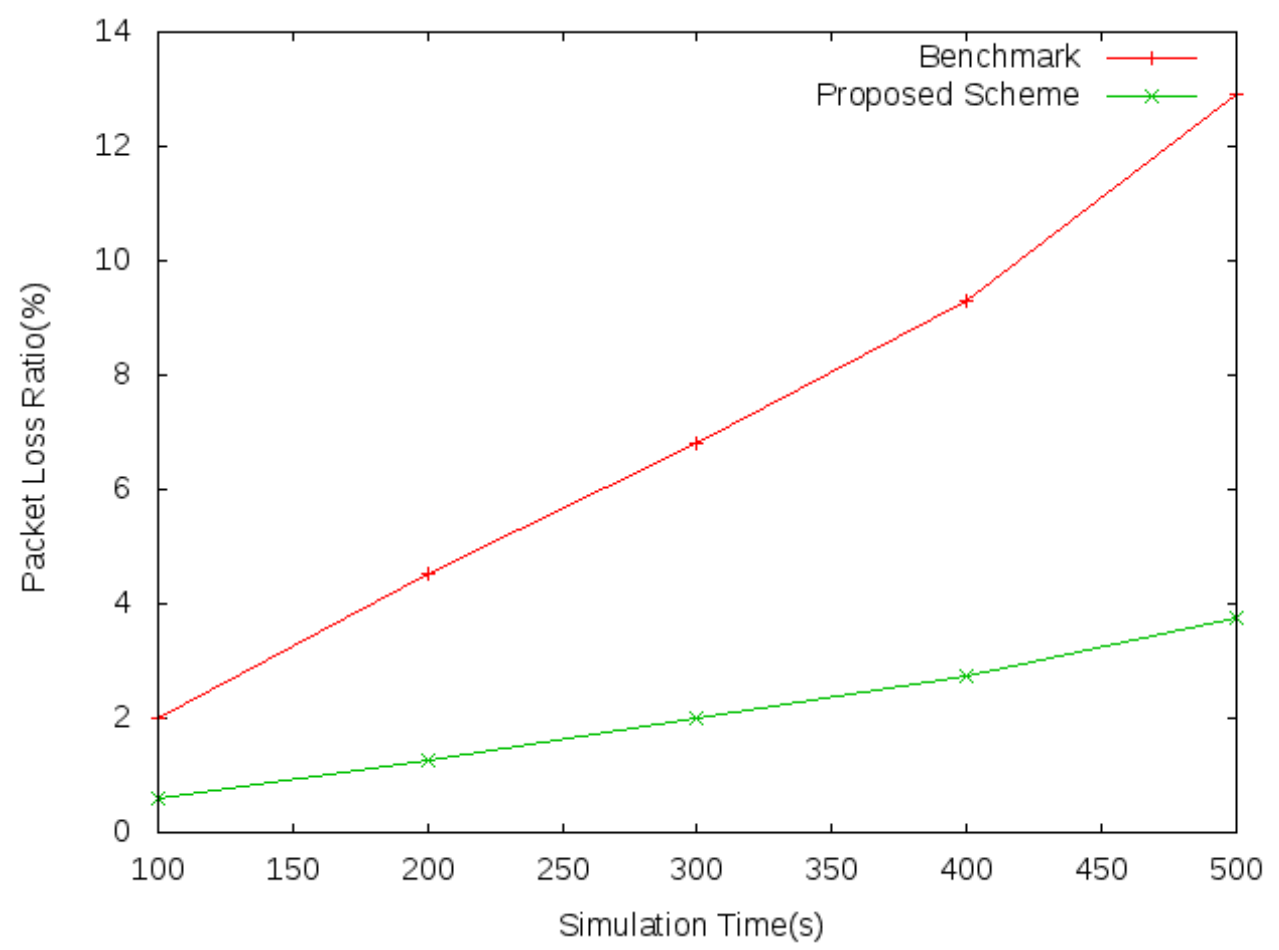

Figure 7. Packet Lost Ratio versus Simulation Time - Comparison 


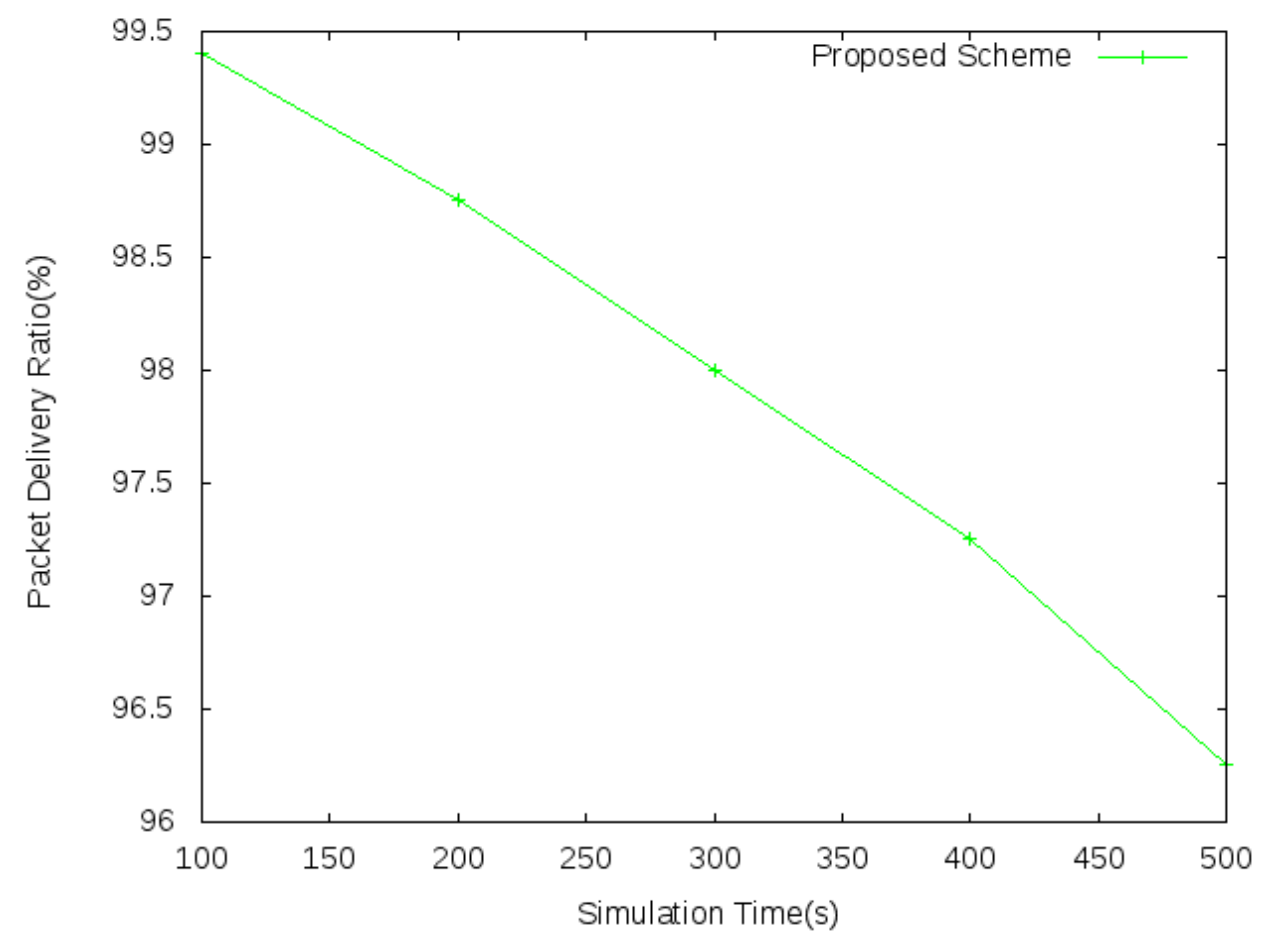

Figure 8. Packet Lost Ratio versus Simulation Time for the Proposed Implementation

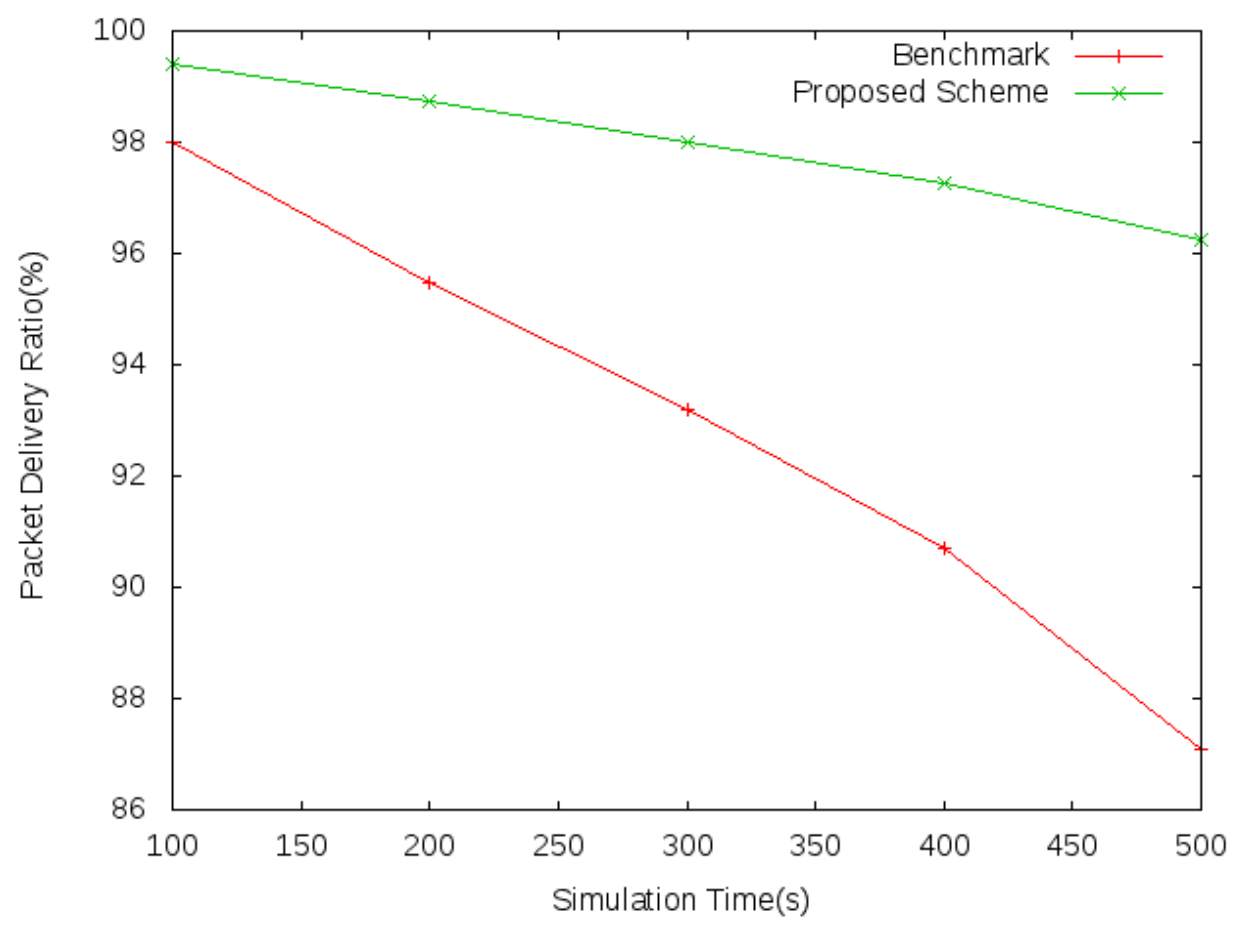

Figure 9. Packet Delivery Ratio versus Simulation Time - Comparison 
When handover latency is reduced, MN does not have to wait for the long handover process configuration setup. Since the MN join the multicast group directly after the MN changes the location. Hence, there will be smooth context delivery with no connection break, and thus traffic can continuously running without any interruption. This has significantly reduced the possibility of packet loss occurrence. Therefore, the number of packet delivery ratio is high as can be seen in Figure 9 and Figure 10.

The effectiveness of the implementation is shown in the Figure 8 and Figure 10. It can be seen that there is huge gap between the improvised implementation and the standard. By using PMIPv6 with CTMFR, the performance for 100 packets is almost more than $50 \%$ better than the standard method. For real-time application like Internet, the occurrence of packet loss is common. The high packet loss rate will seriously degrade the service quality of real-time applications received by MN. In order to avoid large performance fluctuation during handover, MN should experience no significant difference in packet loss rate before and after handover process. Low packet loss rate has been identified as another dimension of requirements in providing better handover performance.

\section{Conclusion}

The PMIPv6 is enabled with multicast fast reroute and content delivery and evaluated using NS3 simulation. The performance of the selected metric is presented, validated and compared to PMIPv6 standard. The improvised implementation provides better packet loss rate. From the simulation results, this verified the combination of CT and MFR provides lesser performance issue as to the general PMIPv6.

\section{References}

[1] Cisco Visual Networking Index: Global Mobile Data Traffic Forecast Update 2014 - 2019, Cisco White Paper, (2015).

[2] Cisco Visual Networking Index: Forecast and Methodology 2014 - 2019, Cisco White Paper, (2015).

[3] Cisco Visual Networking Index: The Zettabyte Era Trends and Analysis, Cisco White Paper, (2015).

[4] T. C. Schmidt, M. Waehlisch and G. Fairhurst, "Multicast mobility in MIPv6: problem statement \& brief survey", RFC 5757, (2010) February.

[5] C. J. Bernardos, M. Calderon and I. Soto, "PMIPv6 and network mobility problem statement", InternetDraft, (2012) March.

[6] D. Johnson, C. Perkins and J. Arkko, "Mobility support in IPv6", RFC 3775, (2004) June.

[7] S. Gundavelli, K. Ed Leung, V. Devarapalli, K. Chowdhury and B. Patil, "Proxy Mobile IPv6 (PMIPv6)", RFC 5213, (2008) August.

[8] D. Kim, W.-S. Lim and Y.-J. Suh, "Multicast extension to Proxy Mobile IPv6 for mobile multicast services", Journal of Computing Science and Engineering, vol. 5, (2011) December, pp. 316-323.

[9] D. von Hugo and H. Asaeda, "Context Transfer Protocol extension for multicast," Internet-Draft, (2013) February.

[10] L. Shrivastava and G. S. Tomar, "A Critical Analysis of Congestion adaptive routing protocols for Mobile Ad hoc Networks", International Journal of Future Generation Communication and Networking, vol. 9, no. 6, (2016), pp. 9-16.

[11] J. Loughney, M. Nakhjiri, C. Perkins and R. Koodli, "Context Transfer Protocol”, RFC 4067, (2005) July.

[12] T.-T. Nguyen and C. Bonnet, "Performance optimization of multicast context delivery in a mobile environment based on PMIPv6", Wireless Communications and Networking Conference (WCNC) IEEE, (2013) April, pp. 1249-1254.

[13] A. Karan, C. Filsfils, I. J. Wijnands and B. Decraene, "Multicast Only Fasr Reroute (MoFRR)", RFC 7431, (2015) August.

[14] T. Schmidt, M. Waehlisch and S. Krishnan, "Base deployment for multicast listener support in Proxy Mobile IPv6 (PMIPv6) domains", RFC 6224, (2011) April.

[15] H.-k. Zhang, J.-f. Guan, H.-C. Zhou and Y. Zhu, "Multicast routing in Proxy MIPv6", (2007).

[16] T. Schmidt, Waehlisch and S. Krishnan, "Base Deployment for Multicast Listener Support in Proxy Mobile IPv6 (PMIPv6) Domains", RFC 6224, (2011) April.

[17] J. Liu and W. Luo, "Routes Optimization for Multicast Sender in Proxy Mobile IPv6 Domain", Standard Track, (2013). 
[18] J. C. Zuniga, L. M. Contreras, C. J. Bernardos, S. Jeon and Y. Kim, "Multicast Mobility Routing Optimizations for Proxy Mobile IPv6", RFC 7028, (2013) September.

[19] S. Singh Kushwah and G. S. Tomar, "Investigation of Effects of Mobility on Routing Protocols in MANET", UCMA, International Conference on Ubiquitous Computing and Multimedia Applications, (2011), pp. 82-84.

[20] L. Wang, S. Goa, H. Zhang, T. C. Schmidt and J. Guang, "Mobile multicast source support in PMIPv6 networks", Journal on Wireless Communications and Networking. (2013) June.

[21] A. Prithiviraj, K. Krishnamoorthy and K. Jaya Bharathy, "Optimizing handover performance for heterogeneous wireless networks in pmipv6", International Journal of Advanced Research in Computer Science, vol. 5, Is. 1, (2014) January-February, pp. 89-92.

[22] B. J. Majerick, G. J. Heijenk, A. Pras and P. T. de Boer, "Lossless Multicast Handovers in Proxy Fast Mobile IPv6 Networks", University of Twente, (2015) February.

[23] F. Abdul Jalin and R. Alsaqour, "A Simulation Study of Proxy Mobile IPV6 (PMIPV6) Protocol", Journal of Engineering and Applied Sciences, vol. 11, no. 7, (2016).

[24] S., Kim, Y., "Deployment Models for Distributed Mobility Management", draft-sijeon-dmmdeployment-models-02 (work in progress), (2016) March. 